\title{
Relación entre bienestar subjetivo, optimismo y variables sociodemográficas en estudiantes universitarios de la Universidad de San Luis Potosí en México*
}

\author{
Relationship between Subjective Well-being, Optimism \\ and Demographic Variables in College Students of \\ the University of San Luis Potosi in Mexico
}

Recibido: julio 17 de 2012 | Revisado: mayo 20 de 2013 | Aceptado: marzo 13 de 2014

\author{
Rosario Josefa MARrero QUeVEdo** \\ Mónica Carballeira Abella \\ Universidad de La Laguna, Tenerife, España \\ José Ángel GonZÁlez Villalobos *** \\ Universidad de San Luis Potosí, México
}

doi:10.11144/Javeriana.UPSY13-3.rbso

Para citar este artículo: Marrero, R. J., Carballeira, M. \& González, J. A. (2014). Relación entre bienestar subjetivo, optimismo y variables sociodemográficas en estudiantes universitarios de la Universidad de San Luis Potosí en México. Universitas Psychologica, 13(3), 1083-1098. http://dx.doi. org/10.11144/Javeriana.UPSY13-3.rbso

\footnotetext{
Artículo de investigación

*** Departamento de Psicología Clínica, Psicobiología y Metodología. Facultad de Psicología. Correos electrónicos: rmarrero@ull.es, mabella@ull.es

***** Correo electrónico: joanviel@ull.es
}

\section{RES UMEN}

El objetivo de este estudio fue analizar la relación entre características sociodemográficas, optimismo y bienestar subjetivo (SWB) en estudiantes universitarios mexicanos de la Universidad de San Luis Potosí. Además, identificar qué áreas vitales incidían en mayor medida en el bienestar global y cuánto aportaría el optimismo a esta relación. Se recogió información de 299 estudiantes (17 a 49 años; $M=19.38 ; D E=3.19$ ) mediante una entrevista semiestructurada, registrando diversas características sociodemográficas que se consideraron relevantes. El optimismo disposicional se midió empleando el Life Orientation Test Revised (Scheier, Carver \& Bridges, 1994) y el bienestar subjetivo mediante diversas medidas: felicidad (Subjective Happiness Scale de Lyubomirsky \& Lepper, 1999), satisfacción vital (Satisfaction with Life Scale de Diener, Emmons, Larsen \& Griffin, 1985), emociones positivas y emociones negativas (Positive and Negative Affect Schedule de Watson, Clark \& Tellegen, 1988) y satisfacción en los dominios vitales de estudios, pareja, salud y ocio (Entrevista semiestructurada de Marrero, Carballeira \& Rodríguez, 2007). A través de la Tau-b de Kendall se analizó la relación de las variables sociodemográficas con el optimismo y los indicadores de bienestar, encontrando asociaciones entre género y satisfacción con el trabajo/estudios, satisfacción vital, felicidad y optimismo. Además, tener una relación sentimental se relacionaba con la satisfacción con la pareja y la condición laboral con satisfacción vital. Los análisis correlacionales de Pearson entre los ocho indicadores de bienestar y el optimismo mostraron que todos los indicadores de bienestar se relacionaban entre ellos y el optimismo estaba asociado en mayor medida que las variables sociodemográficas al bienestar, siendo la relación con medidas globales mayor que con los dominios vitales específicos. Para profundizar en esta asociación entre optimismo y bienestar se dividió a los participantes en tres grupos según su nivel de optimismo. A través de un MANOVA se compararon las medias en bienestar encontrando que las personas con optimismo alto y medio informaron de mayor satisfacción vital y emociones positivas, menor emociones negativas y mayor satisfacción en todos los dominios vitales frente a las personas con bajo optimismo El MANCOVA, tomando el género como covariante, indicaba resultados similares. Por último, se 
aplicaron análisis de regresión múltiple para conocer el peso que tenían las distintas medidas de satisfacción en dominios específicos en los indicadores globales de bienestar, felicidad y satisfacción vital. Los resultados mostraron que todos los dominios son relevantes para ambas variables criterio pero la satisfacción laboral/estudios resultó ser el predictor más poderoso. Cuando se realizaron nuevos análisis de regresión múltiple, incluyendo el optimismo como predictor, se encontró que este explicaba mayor porcentaje de varianza que la satisfacción en dominios específicos, sobre todo en el caso de la felicidad. Estos hallazgos sugieren que el optimismo es un rasgo de personalidad relevante para mejorar el bienestar subjetivo de los individiduos.

Palabras clave autores

optimismo; bienestar subjetivo; felicidad; satisfacción vital; emociones positivas; emociones negativas; satisfacción en dominios vitales específicos

\section{A B S T R A C T}

The main objective of this study was to analyze the relationship between different sociodemographic characteristics, optimism and subjective well-being (SWB) in a sample of Mexican undergraduate students from the University of San Luis Potosi. Additionally, we have tried to identify which specific life domains influenced more on global well-being and how the optimism could explain well-being. Data were collected of 299 participants aged between 17 and 49 years old $(M=19.38 ; \mathrm{SD}=3.19)$ through a semi structured interview, considering different relevant sociodemographic characteristics. Dispositional optimism was evaluated using the Life Orientation Test Revised (Scheier, Carver \& Bridges, 1994). Subjective well-being included the following measures: Happiness (Subjective Happiness Scale de Lyubomirsky \& Lepper, 1999), Life Satisfaction (Satisfaction with Life Scale from Diener, Emmons, Larsen \& Griffin, 1985), Positive and Negative Emotions (Positive and Negative Affect Schedule from Watson, Clark \& Tellegen, 1988) and Satisfaction with different Life Domains: Job/ Studies, Partner, Health and Leisure (Marrero, Carballeira \& Rodríguez, 2007). Through the Test for Kendall's Tau-b, the relationship between sociodemographic variables, optimism and well-being indicators was analysed. The results showed moderate associations between gender and studies satisfaction, life satisfaction, happiness and optimism. Also, the fact of having a sentimental relationship was related to partner satisfaction; and the job situation was associated with life satisfaction. Pearson correlational analyses between the eight well-being indicators and the optimism showed that all the well-being variables were related each other. Optimism was more associated to all the measures of SWB than the sociodemographic variables; and the relationships of optimism with global measures of well-being were greater than those with the specific life domains. In order to deep in this association between optimism and well-being, the sample was split in three groups in function of the level of optimism. A MANOVA was made to compare the means of well-being, finding that those participants with high and medium optimism, vs. those with low optimism, reported more life satisfaction and positive emotions, lower negative emotions and more satisfaction in all the specific life domains. The MANCOVA, taking the gender as a covariant, showed similar findings. Finally, Multiple Regression Analyses were applied to know the influence of the different specific life domains on the global components of well-being: happiness and life satisfaction. The results showed that all the specific domains were relevant for both criteria variables, but studies satisfaction was the more powerful predictor. In the new multiple regression analyses, the optimsm was included and explained a higher percentage of variance than the measures of satisfaction with life domains, especially in the case of happiness as the criteria. These findings suggested that optimism is a relevant personality trait to improve subjective well-being of individuals.

Keywords

optimism; subjective well-being; happiness; life satisfaction; positive emotions; negative emotions; satisfaction with specific life domains

\section{Introducción}

En los últimos años ha habido un creciente interés por estudiar los determinantes positivos del comportamiento humano, surgiendo lo que se ha venido a denominar la Psicología Positiva (Seligman \& Csikszentmihalyi, 2000). En un artículo de revisión, Ryan y Deci (2001) clasificaron los estudios sobre bienestar que existían hasta ese momento, reconociendo dos tradiciones claramente diferenciadas: aquella centrada en el estudio del bienestar subjetivo el bienestar subjetivo o hedónico, entendido como la 'maximización del placer y minimización del dolor' propuesta inicialmente por Bradburn (1969) e impulsada posteriormente por Diener y su equipo (Diener, 1984; Diener, Emmons, Larsen \& Griffin, 1985; Diener, Suh, Lucas \& Smith, 1999); y la centrada en el bienestar psicológico o eudaimónico que se define como la 'búsqueda de una vida virtuosa o de excelencia, en la que predomina el desarrollo de las potencialidades del individuo y la autorrealización' (Ryff, 1989).

Este trabajo se centra en el estudio del bienestar subjetivo analizando los componentes del mismo, así como las variables sociodemográficas y personales que se asocian a este. El bienestar subjetivo se ha entendido con base en los dos componentes propuestos por Diener (1984): el afectivo, referido al predominio de emociones positivas frente a las emociones negati- 
vas y cuyo equilibrio ha venido a denominarse felicidad (Argyle, 1999; Bradburn, 1969; Lyubomirsky \& Lepper, 1999) y el cognitivo, relacionado con la satisfacción vital y que recoge la valoración global que realiza el individuo acerca de los logros obtenidos en su vida en función de sus expectativas o estándares personales (Pavot \& Diener, 1993). Aunque la mayor parte de la investigación se ha centrado en este tipo de medidas globales de bienestar, existen otros indicadores más específicos que evalúan, también desde un punto de vista cognitivo, la satisfacción del individuo en dominios o áreas concretas de su vida tales como salud, productividad, vida privada, seguridad, vida en comunidad, bienestar emocional y bienestar material (Cummings, Eckersley, Pallant \& Davern, 2002). El bienestar subjetivo implica, por tanto, una valoración integral que hace el individuo acerca de sus vivencias, logros, fracasos, emociones, o factores de crianza, entre otros, considerando tanto áreas concretas, como su vida a nivel global y que únicamente se podrá conocer mediante el propio juicio del individuo (Rojas \& Elizondo-Lara, 2012).

Aunque la investigación acerca del bienestar subjetivo y sus determinantes no es reciente (DeNeve \& Cooper, 1998; Diener, 1984; Diener \& et al., 1985; Fordyce, 1986; Pavot \& Diener, 1993; Ryff, 1989), es en la última década en que ha comenzado a estudiarse de manera sistemática en países lationoamericanos (Casullo, 2000; Díaz \& González, 2011; Laca, Verdugo \& Guzmán, 2005; Moyano \& Ramos, 2007; Palomar, 2000; PuenteDíaz \& Cavazos, 2013; Vera-Noriega \& Tánori, 2002). Según Castro (2012) esto ha supuesto el $5 \%$ del total de publicaciones sobre Psicología Positiva que aparecen en las principales revistas latinoamericanas desde el 2000 hasta el 2012, proviniendo la mayor parte de los artículos (el 80\%) de solo cuatro países: México, Chile, Brasil y Argentina. Y centrándose estos estudios, por un lado, en analizar las áreas o dominios vitales que están más relacionados con el bienestar (Palomar, 2004; Rojas \& Elizondo-Lara, 2012; Vera-Noriega \& Rodríguez, 2007; Vera-Noriega \& Tánori, 2002) y por otro, en identificar las características sociodemográficas que influyen fundamentalmente en la satisfacción vital (Fuentes \& Rojas, 2001; Moyano \& Ramos, 2007;
Ruvalcaba, Salazar \& Fernández-Berrocal, 2012; Vera-Noriega, 2001).

Los hallazgos acerca de los dominios vitales que contribuyen al bienestar de los mexicanos indican que las áreas de la familia, tanto las relaciones con la pareja como con los hijos, el gobierno y la satisfacción global con la vida se presentan como los factores más importantes del bienestar subjetivo (Palomar, 2004; Vera-Noriega \& Tánori, 2002). Además, también aparecen los amigos y el área personal como determinantes de la satisfacción vital y la felicidad, sobre todo en población estudiante (Arita, 2005; Banda \& Morales, 2012; Vera-Noriega, 2001; Vera-Noriega \& Rodríguez, 2007). De modo que, existe cierto acuerdo en que la satisfacción con la vida parece depender en mayor medida de áreas como la familiar, laboral y tiempo libre (Rojas \& Elizondo-Lara, 2012).

No obstante, algunos de estos resultados parecen estar modulados por características sociodemográficas, como el género, la edad, el estado civil o los ingresos. En general, las mujeres parecen mostrar mayor bienestar o satisfacción con la vida que los hombres (Domínguez et al., 2006; Laca \& et al., 2005; Vera-Noriega \& Rodríguez, 2007), pero va a depender del dominio vital que se tome en consideración. Los hombres informan de más satisfacción en dominios como amigos, área personal y área económica (Vera-Noriega, 2001). En un estudio reciente, llevado a cabo en Costa Rica, las mujeres mostraron mayor satisfacción en el dominio familia extensa y amistades, pero menor satisfacción en el dominio económico (Rojas \& Elizondo-Lara, 2012). Por lo anterior, la investigación previa muestra ciertas inconsistencias en cuanto a la influencia del género en el bienestar.

Los estudios que analizan el bienestar en función de la edad muestran una relación en forma de "U" invertida. De modo que los jóvenes y los mayores son los que informan de mayor satisfacción con la vida y más emociones positivas, aunque parece que la expresión de emociones y la percepción social de la felicidad disminuye en los participantes de más edad (Vera-Noriega \& Rodríguez, 2007). Nuevamente, dependerá del dominio vital que se analice, pero parece que la satisfacción con el do- 
minio familiar es importante para la mayor parte de los individuos siendo bastante estable con los años (Rojas \& Elizondo-Lara, 2012).

El estado civil también se ha vinculado a la felicidad, informando las personas casadas de más felicidad que las solteras, separadas o viudas (Argyle, 1999; Lu, 2000). En adultos peruanos se ha encontrado que las personas casadas informaron de mayor felicidad que las solteras (Alarcón, 2001). Según Namkee y Mochón (2007), la pareja es importante no solo por proporcionar estabilidad emocional sino que satisface otras áreas vitales como la sexual y el ocio.

Por otra parte, existe cierta controversia acerca de la importancia de los aspectos económicos en el bienestar, aunque parece un área relevante no afecta del mismo modo a individuos de distintas edades, siendo en algunos casos más importante para el bienestar de los jóvenes que de los mayores (Palomar, 2000) y, en otros, no apareciendo diferencias en satisfacción general con la vida entre jóvenes que tienen distintas condiciones de vida, incluso de extrema pobreza (Ruvalcaba et al., 2012). Algunos autores señalan que los ingresos solo explicarían el 5\% de la varianza de bienestar, siendo más importante la percepción de necesidades materiales satisfechas (Fuentes \& Rojas, 2001).

Por último, hay que considerar que en Latinoamérica solo algunos estudios se han centrado en identificar factores psicológicos, como el desarrollo de la identidad, la autonomía, rasgos personales o estrategias de afrontamiento que repercuten de algún modo en la satisfacción vital y la felicidad (Domínguez et al., 2006; Escobar, Zanatta, Ponce, García \& Gama, 2012; Puente-Díaz \& Cavazos, 2013; Verdugo-Lucero et al., 2013).

Atendiendo a las inconsistencias encontradas en cuanto a la relación entre características sociodemográficas y bienestar, considerado tanto a nivel global como en dominios vitales específicos, y al escaso consenso acerca del peso que tiene cada área vital en el bienestar, surge el presente estudio. Además, teniendo en cuenta que la investigación previa ha mostrado la relevancia de determinadas características personales en el bienestar (Brissette, Scheier \& Carver, 2002; Chico, 2002; Marrero \& Carballeira, 2011; Steel, Schmidt \& Shultz, 2008), pero que ape- nas han sido estudiadas con población latinoamericana, se analiza el papel que tiene el optimismo en los distintos componentes del bienestar, entendido como una expectativa global o generalizada de que en el futuro se obtendrán resultados favorables frente a los desfavorables (Scheier \& Carver, 1985). Por tanto, los objetivos fundamentales de este trabajo son: 1. Analizar la relación entre características sociodemográficas y los distintos componentes del bienestar subjetivo (satisfacción vital, felicidad, emociones positivas, emociones negativas y satisfacción en los dominios de pareja, estudios, salud y ocio); 2. Analizar la relación entre el optimismo y los componentes del bienestar subjetivo, así como el nivel de bienestar experimentado por las personas con alto optimismo; 3. Analizar el peso que tienen tanto los distintos dominios vitales como el optimismo en la satisfacción y la felicidad de los individuos.

\section{Método}

\section{Participantes}

Participaron 299 estudiantes de la Universidad de San Luis Potosí (UASLP) del primer curso de Psicología, contactados a solicitud de sus docentes para colaborar voluntariamente en una investigación acerca del bienestar y sus determinantes. Las edades estuvieron comprendidas entre los 17 y los 49 años $(M=19.38$; $D E=3.19)$; el $71.8 \%$ eran mujeres y el resto hombres. El $94.9 \%$ era soltero y una mínima parte estaba casado o convivía con su pareja (5.1\%). El 37.1\% del total mantenía una relación sentimental, mientras que la mayoría informaba de no tener pareja. Únicamente el 3.1\% estaba activo laboralmente, aunque la mayor parte era estudiante y no tenía trabajo remunerado, el 64.7\% informaba que el dinero del que disponía le permitía tener sus necesidades cubiertas.

\section{Instrumentos}

Entrevista semiestructurada (Marrero, Carballeira Eु Rodríguez, 2007)

Fue diseñada por el equipo de investigación, con el propósito de registrar diversas características 
sociodemográficas y de estilo de vida de los participantes. Consta de siete apartados amplios: 1) datos generales: donde se recoge el género, la edad, el lugar de residencia, el estado civil, el número de hijos y el nivel de satisfacción con la situación sentimental actual, esta última en una escala tipo Likert desde 1 (nada satisfecho) hasta 5 (muy satisfecho); 2) estudios y profesión: donde se registra el nivel de estudios alcanzado (sin estudios, estudios primarios, estudios secundarios, bachiller o estudios universitarios), la profesión, el estado laboral actual (activo, parado, jubilado o estudiante), la satisfacción con su trabajo o estudios (de 1 a 5 puntos), los ingresos económicos y si estos le permiten cubrir sus necesidades, respondiendo, en este último caso, afirmativa o negativamente; 3) salud: sondea si existe algún tipo de enfermedad física y otros hábitos de salud, tales como consumo de tabaco, alcohol, drogas o si practica algún deporte, en este apartado también se pregunta acerca del nivel de satisfacción general con su salud en la misma escala de cinco puntos; 4) creencias religiosas: donde se registra si tiene alguna educación religiosa, de qué tipo, así como la importancia de la religión en su vida; 5) historia familiar: se recoge el estado civil de los padres y diversos adjetivos que definen la relación con su madre y con su padre; 6) ocio y diversión: donde aparece un listado de actividades para que el individuo registre la periodicidad con que realiza las mismas (desde nada hasta a diario) y el nivel de satisfacción con las actividades lúdicas en una escala de cinco puntos (desde nada satisfecho hasta muy satisfecho) y, por último, 7) acontecimientos vitales: donde se plantean tres cuestiones abiertas acerca de los acontecimientos importantes ocurridos en su vida, los hechos que resultaron más agradables y aquellos que fueron más desagradables.

En este trabajo, únicamente, se consideraron las variables sociodemográficas: género, edad, estado civil, situación de pareja, nivel de estudios, nivel suficiente de ingresos y situación laboral; así como el nivel de satisfacción en cuatro áreas vitales: pareja, trabajo/estudio, salud y ocio, que tal y como se señaló anteriormente fueron evaluadas mediante una escala tipo Likert de 1 a 5 puntos $(1=$ no estoy satisfecho/a; 2 = algo satisfecho/a; 3 = medianamente satisfecho/a; $4=$ bastante satisfecho/a; $5=$ muy satisfecho/a). Los participantes, al final de la entrevista tenían un apartado de observaciones para señalar si se encontraron con dificultades en la comprensión de los ítems. No habiendo ninguna observación al respecto, se consideró que la entrevista era adecuada para registrar las variables objeto de estudio.

Life Orientation Test Revised ([LOT-R];

Scheier, Carver Eु Bridges, 1994)

Incluye 6 ítems y otros 4 neutros, con cinco opciones de respuesta, que permite evaluar el optimismo disposicional o las expectativas de los individuos acerca de los resultados favorables que puedan ocurrirles en el futuro (p. ej.: "En los momentos de incertidumbre, suelo esperar lo mejor"). En el estudio original, la prueba presentó una consistencia interna de 0.78 . Este instrumento ha mostrado ser adecuado para su utilización con población mexicana (Vera-Villarroel, Córdova-Rubio \& Celis-Atenas, 2009). En este estudio, se empleó la traducción al español, realizada por el equipo de investigación, siendo el alpha de Cronbach de 0.57.

Satisfaction with Life Scale

([SWLS]; Diener et al., 1985)

Mediante cinco ítems, se evalúa el juicio cognitivo del individuo acerca de la satisfacción global con su propia vida, comparando sus circunstancias vitales con un estándar particular (p. ej.: "En muchos sentidos, mi vida está cercana a lo ideal”). Cada ítem se responde en una escala de 1 (nada satisfecho) a 7 (muy satisfecho). La consistencia interna de la escala original fue de 0.87 y la fiabilidad test-retest 0.82. En este trabajo, también se utilizó la versión española traducida por el equipo de investigación que coincide con la versión adaptada a población mexicana realizada por Laca et al. (2005), mostrando una consistencia interna de 0.84 .

Positive and Negative Affect Schedule ([PANAS]; Watson, Clark 8 Tellegen, 1988)

Consta de 20 calificativos, 10 positivos (p. ej.: entusiasmado, motivado, decidido) y otros 10 negativos 
(p. ej.: inquieto, nervioso, temeroso) que evalúan las emociones presentes en el momento de contestar la prueba, en una escala que oscila desde 0 (totalmente en desacuerdo) a 5 (totalmente de acuerdo). Se empleó la versión traducida y adaptada al español por el equipo de investigación, en la que se aclararon con jueces expertos de procedencia mexicana, la mitad de emociones registradas que parecían ser confusas. En este estudio, los índices de consistencia interna de la prueba, tal y como se adaptó en los distintos calificativos, fueron 0.82 para emociones positivas y 0.85 para emociones negativas.

\section{Subjective Happiness Scale \\ (Lyubomirsky Ë Lepper, 1999)}

Está formada por 4 ítems que evalúan, en una escala de 7 puntos, el grado en que el individuo se siente feliz en relación con su vida o comparado con las personas de su entorno (p. ej.: "En general me considero una persona feliz"). La escala en el estudio original de validación ha mostrado coeficientes de fiabilidad que oscilan entre 0.79 y 0.94 . En este estudio, en el cual se empleó la versión española traducida por el equipo de investigación, la consistencia interna fue de 0.81 .

\section{Procedimiento}

Se contactó con el profesorado de primero de Psicología de la UASLP para que facilitara un tiempo de 30 minutos a su alumnado en el que pudieran cumplimentar la batería de pruebas a través de una aplicación en línea. En la prueba se explicaban brevemente los objetivos de la investigación y se garantizaba la confidencialidad de la información recabada. Además, se aclaraba que la información sería utilizada con fines de investigación, por lo que la cumplimentación de la misma supondría el consentimiento de los participantes para emplear los datos en publicaciones científicas.

\section{Análisis de datos}

Se empleó un diseño descriptivo transversal o exploratorio incorporando los datos obtenidos al paquete estadístico SPSS (versión 19), a través del cual se llevaron a cabo diversos análisis estadísticos. En todos los análisis se optó por la estadística paramétrica, a pesar de que la muestra fue de conveniencia y no estaba aleatorizada, pero las variables que se incluyeron en el estudio, a priori, se distribuían normalmente y el objetivo último era estimar un parámetro de interés, en este caso el bienestar, a partir de la combinación de diversas variables, lo que no era posible mediante técnicas no paramétricas.

En un primer momento, con el objeto de conocer la relación entre las variables de bienestar, las características sociodemográficas y el optimismo se emplearon análisis correlacionales. Por un lado, se aplicó la Tau-b de Kendall entre los indicadores de bienestar, el optimismo y las variables sociodemográficas ordinales o categóricas (género, estado civil, relación sentimental, estudios, ingresos y condición laboral). Por otra parte, se aplicó correlación de Pearson entre las variables continuas que en este estudio fueron la edad, los indicadores de bienestar y el optimismo. Teniendo en cuenta que el optimismo guardaba una relación estrecha con el bienestar, se profundizó en el papel del mismo, analizando si existían diferencias en las medias de bienestar de los individuos en función de que su nivel de optimismo fuera bajo, medio o alto, para cuyo análisis se empleó un MANOVA puesto que permite comparar a los tres grupos simultáneamente, así como incluir al mismo tiempo diversas variables dependientes.

Previamente, se había comprobado, mediante $\chi^{2}$, que los grupos fueran homogéneos en las variables sociodemográficas registradas. $\mathrm{Al}$ aparecer diferencias entre los tres grupos de optimismo en género, se aplicó MANCOVA, en el que se incluyó el género como covariante. Por último, para averiguar qué dominio vital explicaba en mayor medida el bienestar global (satisfacción con la vida y felicidad), se aplicaron análisis de regresión múltiple con el método hacia adelante que permite conocer qué variables van a formar parte de la ecuación en función del orden de importancia. Las variables predictoras fueron la satisfacción en las cuatro áreas vitales y como variables criterio se tomaron la satisfacción vital, por un lado, y la felicidad, por 
otro. Seguidamente, se aplicaron nuevos análisis de regresión, con el objeto de identificar la importancia del optimismo en el bienestar frente a la satisfacción en dominios vitales. En esta ocasión se incluyeron como variables predictoras, conjuntamente, las cuatro medidas de satisfacción en áreas vitales y el optimismo.

\section{Resultados}

En un primer momento, se analizó la distribución de las puntuaciones en las distintas variables de bienestar. En general, se observa una distribución normal en las variables emociones positivas $(M=$ 38.66; $D E=5.83)$ y emociones negativas $(M=$ 21.05; $D E=6.2$ ), pero hay una cierta asimetría, situándose la mayor parte de los participantes por encima de la media, en felicidad $(\mathrm{M}=22.91 ; \mathrm{DE}$ =3.91); satisfacción vital $(M=28.20 ; \mathrm{DE}=6.2)$ y, sobre todo, en la satisfacción con los dominios vitales específicos. El 75.5\% informa de niveles de satisfacción muy altos en estudios $(M=3.97 ; \mathrm{DE}$ $=0.73)$; el 74.6\% muestra niveles muy altos de satisfacción con la salud ( $\mathrm{M}=3.91 ; \mathrm{DE}=0.8)$; el $66.1 \%$ tiene una alta satisfacción con el ocio $(\mathrm{M}=$ 3.84; $D E=0.88)$ y un $60.2 \%$ está muy satisfecho con su pareja $(M=3.64 ; D E=1.07)$, en este último caso hay que tener en cuenta que solo algo más del tercio tenía pareja.

Los análisis correlacionales, Tau-b de Kendall y Pearson, indican que apenas existe relación entre las características sociodemográficas y bienestar subje- tivo. Tal y como se puede observar en la Tabla 1 , no aparecen relaciones significativas del bienestar, evaluado de modo global y específico, con edad, estado civil, nivel de estudios, ni ingresos. El género muestra una correlación de baja magnitud con satisfacción en los estudios $(r=0.14 ; p<0.01)$, satisfacción vital $(r=0.14 ; p<0.01)$, felicidad $(r=0.14 ; p<0.01)$ y optimismo $(r=0.11 ; p<0.05)$. Tener pareja se relaciona con la satisfacción en el área sentimental $(r=-0.31 ; p<0.001)$, y la condición laboral está ligeramente asociada a la satisfacción vital $(r=0.12$; $p<0.01)$. Cuando se analizan, a través de un MANOVA, las diferencias entre hombres y mujeres en las medidas de bienestar que aparecen relacionadas con el género, se observa que las mujeres informan de mayor satisfacción con los estudios $(F(1,286)=$ $6.09 ; p<0.01)$; mayor felicidad $(F(1,286)=9.93 ; p$ $<0.01)$; mayor satisfacción con la vida $(F(1,286)=$ $10.2 ; p<0.01)$ y mayor optimismo $(F(1,286)=5.98$; $p<0.01)$. Un ANOVA permitió analizar a las personas que tiene pareja frente a las que no la tienen en la variable satisfacción con la pareja, indicando que las personas que tienen pareja están más satisfechas con su relación que los que no la tienen $(F(1,269)$ $=30.84 ; p<0.001)$. Se aplicó otro ANOVA para la condición laboral, tomando como variable dependiente la satisfacción vital, se encontró que los estudiantes informan de más satisfacción vital que las personas activas laboralmente, aunque estas diferencias no resultaron significativas $(F(3,291)=$ $1.94 ; p=0.12$ ).

TABLA 1

Correlación entre bienestar subjetivo y características sociodemográficas

\begin{tabular}{|c|c|c|c|c|c|c|c|}
\hline & Género $^{a}$ & $\mathrm{Edad}^{\mathrm{b}}$ & $\begin{array}{l}\text { Estado }^{a} \\
\text { Civil }\end{array}$ & $\begin{array}{c}\text { Relación }{ }^{\mathrm{a}} \\
\text { sentimental }\end{array}$ & Estudios $^{\mathrm{a}}$ & $\begin{array}{l}\text { Ingresos }^{\mathrm{a}} \\
\text { Suficientes }\end{array}$ & $\begin{array}{c}\text { Condición } \\
\text { laboral }^{\mathrm{a}}\end{array}$ \\
\hline Felicidad & $0.14 * *$ & 0.01 & 0.01 & -0.02 & -0.04 & -0.11 & 0.09 \\
\hline Satisfacción vital & $0.14 * *$ & -0.01 & -0.009 & -0.03 & -0.007 & -0.04 & $0.12 * *$ \\
\hline Emociones positivas & -0.04 & 0.05 & -0.003 & 0.02 & -0.07 & -0.04 & 0.04 \\
\hline Emociones negativas & -0.06 & -0.05 & -0.005 & -0.03 & 0.03 & 0.05 & -0.04 \\
\hline Satisfacción pareja & 0.07 & 0.04 & 0.06 & $-0.31 * * *$ & 0.02 & -0.14 & 0.07 \\
\hline Satisfacción estudios & $0.14 * *$ & 0.06 & 0.07 & 0.02 & -0.08 & -0.03 & 0.02 \\
\hline Satisfacción salud & -0.03 & 0.01 & -0.04 & 0.06 & -0.08 & -0.06 & 0.09 \\
\hline Satisfacción ocio & -0.08 & 0.1 & -0.04 & 0.07 & 0.009 & -0.01 & -0.07 \\
\hline Optimismo & $0.11 *$ & -0.006 & -0.06 & 0.004 & -0.06 & -0.06 & 0.008 \\
\hline
\end{tabular}

$* p<0.05 . * * p<0.01 . * * * p<0.001$. a: Correlación de Tau-b de Kendall; b: Correlación de Pearson.

Fuente: elaboración propia 
En cuanto a la relación entre las distintas medidas de bienestar subjetivo, en la Tabla 2 se observa que las medidas globales de bienestar (satisfacción vital y felicidad) están más relacionadas con los componentes afectivos (emociones positivas y negativas) del bienestar que las medidas de satisfacción en áreas vitales específicas. La felicidad se relaciona de forma directa con emociones positivas $(r=0.62$; $p<0.001)$ e inversamente con emociones negativas $(r=-0.44 ; p<0.001)$; y un patrón similar aparece entre satisfacción vital y emociones positivas $(r=$ $0.47 ; p<0.001)$ y emociones negativas $(r=-0.32$; $p<0.001$ )

La satisfacción en áreas vitales específicas guarda una relación moderada con emociones positivas y de baja magnitud con las emociones negativas. Así, por ejemplo mientras la satisfacción con los estudios y con el ocio se relaciona con emociones positivas $(r=0.37 ; p<0.001 ; r=0.34 ; p<0.001$, respectivamente), muestran una escasa asociación con emociones negativas $(r=-0.17 ; p<0.01 ; r=$ $-0.22 ; p<0.001$, respectivamente). La satisfacción con la relación sentimiental es el área que está más relacionada con la satisfacción vital y la felicidad $(r$ $=0.34 ; p<0.001 ; r=0.32 ; p<0.001$, respectivamente). Por otra parte, la felicidad y la satisfacción vital, aunque guardan una estrecha relación $(r=$ $0.6 ; p<0.001$ ), parecen referirse a constructos ligeramente distintos, compartiendo únicamente el $36 \%$ de la varianza.

En cuanto al optimismo, muestra asociaciones más potentes con todos los indicadores de bienestar que las variables sociodemográficas o que las distintas medidas de satisfacción en áreas vitales. De modo que se relaciona positivamente con felicidad $(r=0.54 ; p<0.001)$, con emociones positivas $(r=$ $0.51 ; p<0.001)$ y con satisfacción vital $(r=0.34$; $p<0.001)$ e inversamente con emociones negativas $(r=-0.33 ; p<0.001)$. Las correlaciones del optimismo con la satisfacción en dominios vitales son de menor magnitud, oscilando entre $0.16(p<$ $0.01)$ y $0.21(p<0.001)$.

Se profundizó en la relación entre optimismo y bienestar, analizando las diferencias entre los individuos en función de su nivel de optimismo. Para ello, se conformaron tres grupos: el grupo de bajo optimismo que se situaba en el centil 25 con puntuaciones entre 1 y 15 (30.4\% de la muestra); el grupo de optimismo medio, con puntuaciones entre 16 y 18 (35.5\% de la muestra) y el grupo de alto optimismo que estaba en el centil 75 y obtuvo puntuaciones de 19 a 24 (34.1\% de la muestra). A

TABLA 2

Correlación de Pearson entre las medidas de bienestar subjetivo y optimismo

\begin{tabular}{|c|c|c|c|c|c|c|c|c|}
\hline & $\begin{array}{l}\text { Satisfacción } \\
\text { vital }\end{array}$ & $\begin{array}{c}\text { Emociones } \\
\text { positivas }\end{array}$ & $\begin{array}{c}\text { Emociones } \\
\text { negativas }\end{array}$ & $\begin{array}{l}\text { Satisfacción } \\
\text { pareja }\end{array}$ & $\begin{array}{l}\text { Satisfacción } \\
\text { estudios }\end{array}$ & $\begin{array}{l}\text { Satisfacción } \\
\text { salud }\end{array}$ & $\begin{array}{l}\text { Satisfacción } \\
\text { ocio }\end{array}$ & Optimismo \\
\hline Felicidad & $0.6 * * *$ & $0.62 * * *$ & $-0.44 * * *$ & $0.32 * * *$ & $0.37 * * *$ & $0.26 * * *$ & $0.29 * * *$ & $0.54 * * *$ \\
\hline $\begin{array}{c}\text { Satisfacción } \\
\text { Vital }\end{array}$ & & $0.47 * * *$ & $-0.32 * * *$ & $0.34 * * *$ & $0.35 * * *$ & $0.24 * * *$ & $0.28 * * *$ & $0.34 * * *$ \\
\hline $\begin{array}{l}\text { Emociones } \\
\text { Positivas }\end{array}$ & & & $-0.3 * * *$ & $0.13^{*}$ & $0.37 * * *$ & $0.28 * * *$ & $0.34 * * *$ & $0.51^{* * * *}$ \\
\hline $\begin{array}{l}\text { Emociones } \\
\text { Negativas }\end{array}$ & & & & $-0.16 * *$ & $-0.17 * *$ & $-0.25 * * *$ & $-0.22 * * *$ & $-0.33 * * *$ \\
\hline $\begin{array}{l}\text { Satisfacción } \\
\text { Pareja }\end{array}$ & & & & & $0.2 * * *$ & $0.14^{*}$ & $0.19 * * *$ & $0.16^{* *}$ \\
\hline $\begin{array}{l}\text { Satisfacción } \\
\text { Estudios }\end{array}$ & & & & & & $0.27 * * *$ & $0.17 * *$ & $0.2 * * *$ \\
\hline $\begin{array}{l}\text { Satisfacción } \\
\text { Salud }\end{array}$ & & & & & & & $0.17 * *$ & $0.21 * * *$ \\
\hline $\begin{array}{l}\text { Satisfacción } \\
\text { Ocio }\end{array}$ & & & & & & & & $0.2 * * *$ \\
\hline
\end{tabular}

$* p<0.05 . * * p<0.01 . * * * p 0.001$.

Fuente: elaboración propia 
través de chi cuadrado se comprobó que los grupos fueron homogéneos en edad $\left(\chi^{2}(2)=24.36, p=\right.$ $0.555)$, estado civil $\left(\chi^{2}(4)=2.04, p=0.729\right)$, nivel de estudios $\left(\chi^{2}(2)=2.93, p=0.318\right)$, ingresos $\left(\chi^{2}(2)=3.55, p=0.169\right)$, condición laboral $\left(\chi^{2}(6)\right.$ $=4.61, p=0.594)$ y relación sentimental $\left(\chi^{2}(2)=\right.$ $4.73, p=0.094)$. Únicamente aparecen diferencias significativas en género $\left(\chi^{2}(2)=5.88, p=0.053\right)$. Las mujeres en términos generales presentan mayor optimismo que los hombres $(F(1,296)=5.29$; $p<0.05)$. Estas diferencias aparecen en el grupo de bajo optimismo, mientras que en el grupo de alto optimismo son los hombres más optimistas que las mujeres.

Tras comprobar la igualdad de las matrices de covarianza entre los tres grupos ( $\mathrm{M}$ de Box $=91.34, p=0.11$ ) y la igualdad de las varianzas de error mediante la prueba de Levene, en la que únicamente había diferencias significativas para felicidad $(F(2,255)=5.26 ; p=0.006$, se aplicó un MANOVA. Se consideraron como variables dependientes las medidas de bienestar y como variable independiente el nivel de optimismo, apareciendo diferencias significativas en todas las medidas de bienestar (Lambda de Wilks $=0.28 ; p<0.001$ ).

Los análisis univariados indican diferencias en felicidad $(F(2,255)=35.45 ; p<0.001 ; \eta 2=0.22)$, satisfacción vital $(F(2,255)=11.81 ; p<0.001 ; \eta 2$ $=0.08)$, emociones positivas $(F(2,255)=31.02$; $p<0.001 ; \eta 2=0.2)$ y emociones negativas $(F(2,255)=9.01 ; p<0.001 ; \eta 2=0.07)$; y en todas las relativas a satisfacción con dominios espe- cíficos: estudios $(F(2,255)=5.01 ; p<0.05 ; \eta 2=$ $0.04)$, pareja $(F(2,255)=4.07 ; p<0.05 ; \eta 2=0.03)$, ocio $(F(2,255)=4.95 ; p<0.01 ; \eta 2=0.04)$ y salud $(F(2,255)=6.18 ; p<0.01 ; \eta 2=0.05)$.

Los análisis post hoc con ajuste de Bonferroni indican que las personas con bajo optimismo muestran menor satisfacción vital, menos emociones positivas, son más infelices y experimentan más emociones negativas que las personas con un nivel de optimismo medio y alto. Además, también se encontraron diferencias significativas entre las personas con optimismo medio y alto en felicidad. En cuanto a la satisfacción en dominios vitales específicos, se observa que las personas poco optimistas están más insatisfechas en todas las áreas vitales que las que tienen puntuaciones altas en optimismo. Únicamente, se encuentran diferencias significativas entre el grupo que presenta bajas puntuaciones en optimismo y el grupo que tiene puntuaciones medias en satisfación con los estudios y satisfacción con la salud (Tabla 3).

Se aplicó un nuevo análisis de varianza tomando como covariante el género (MANCOVA), ya que en los análisis previos se habían observado diferencias entre hombres y mujeres en optimismo. Los resultados van en la misma línea: existen diferencias significativas entre los grupos de optimismo en todas las medidas de bienestar (Lambda de Wilks $=0.89 ; p<0.001)$. A nivel univariado, se observan diferencias en felicidad $(F(2,254)=32.41$; $p<0.001 ; \eta 2=0.2)$, satisfacción vital $(F(2,254)=$ $10.74 ; p<0.001 ; \eta 2=0.08)$, emociones positivas

TABLA 3

Diferencias de medias en función del nivel de optimismo para las medidas de bienestar subjetivo

\begin{tabular}{|c|c|c|c|c|c|}
\hline & $\begin{array}{c}\text { Bajo Optimismo } \\
\text { Media DE } \\
N=81\end{array}$ & $\begin{array}{c}\text { Optimismo Medio } \\
\text { Media DE } \\
N=90\end{array}$ & $\begin{array}{l}\text { Alto Optimismo } \\
\text { Media DE } \\
N=80 \\
\end{array}$ & F & Contraste Bonferroni \\
\hline Felicidad & 20.254 .11 & 23.233 .4 & 24.783 .04 & $35.21 * * *$ & $1<2 * * * ; 1<3 * * * ; 2<3 * *$ \\
\hline Satisfacción vital & 25.95 .24 & 28.285 .12 & 29.844 .98 & $11.81 * * *$ & $1<2 * * ; 1<3 * * *$ \\
\hline Emociones positivas & 34.695 .84 & 39.214 .98 & 40.965 .09 & $29.39 * * *$ & $1<2 * * * ; 1<3 * * *$ \\
\hline Emociones negativas & 23.526 .23 & 21.115 .74 & 19.66 .1 & $9.08 * * *$ & $1<2 * ; 1<3 * * *$ \\
\hline Satisfacción pareja & 3.391 .07 & 3.681 .1 & 3.851 .02 & $3.72 *$ & $1<3^{*}$ \\
\hline Satisfacción estudios & 3.750 .77 & 4.070 .68 & 4.050 .71 & $4.52 * *$ & $1<2 * ; 1<3^{*}$ \\
\hline Satisfacción salud & 3.640 .87 & 3.980 .67 & 40.88 & $6.18 * *$ & $1<2^{* *} ; 1<3 * *$ \\
\hline Satisfacción ocio & 3.640 .82 & 3.790 .92 & 4.070 .88 & $4.95 * *$ & $1<3^{* *}$ \\
\hline
\end{tabular}

$* p<0.05 ; * *<<0.01 ; * * * p<0.001 ; 1=$ Bajo optimismo, $2=$ Optimismo medio; $3=$ Alto optimismo Fuente: elaboración propia 
$(F(2,254)=30.06 ; p<0.001 ; \eta 2=0.19)$, emociones negativas $(F(2,254)=8.43 ; p<0.001 ; \eta 2$ $=0.06)$, satisfacción con los estudios $(F(2,254)=$ $3.62 ; p<0.05 ; \eta 2=0.03)$, satisfacción con la pareja $(F(2,254)=3.22 ; p<0.05 ; \eta 2=0.02)$, satisfacción con el ocio $(F(2,254)=5.19 ; p<0.01 ; \eta 2=0.04)$ y satisfacción con la salud $(F(2,254)=6.72 ; p<$ $0.001 ; \eta 2=0.05)$.

Por último, con el objeto de conocer qué peso o importancia relativa tenían los distintos dominios de satisfacción en cada uno de los indicadores globales de bienestar, se aplicaron análisis de regresión múltiple, empleando el método hacia adelante que permite establecer la combinación lineal de los dominios para explicar la satisfacción vital y la felicidad. Los análisis correlacionales previos revelaron que no existía multicolinealidad entre las distintas variables por lo que fue factible aplicar este estadístico. Las variables predictoras introducidas fueron las cuatro medidas de satisfacción en dominios vitales específicos: pareja, estudios, salud y ocio; y las variables criterio fueron la satisfacción vital en uno de los análisis, y la felicidad en el otro. Tal y como se puede observar en la Tabla 3, los cuatro dominios explican el $25.2 \%$ de la varianza de la satisfacción vital $\left(F(4,253)=22.66, p<0.001, R^{2}\right.$ ajustado $=0.25)$. El área que aparece con más peso es la satisfacción con los estudios $(\beta=0.25, p<$ $0.001)$, seguida de la satisfacción con la pareja $(\beta=$ $0.22, p<0.001)$, con el ocio $(\beta=0.18, p<0.001)$ y con la salud $(\beta=0.14, p<0.05)$. En el caso de la felicidad los resultados son similares, los cuatro dominios explican el $25.8 \%$ de la varianza $(F(4,253)$ $=23.33 p<0.001, \mathrm{R}^{2}$ ajustado $\left.=0.26\right) \mathrm{y}$ de nuevo la satisfacción con los estudios tiene mayor poder predictivo seguida de la satisfacción con la pareja, con el ocio y con la salud.

Seguidamente, se aplicaron nuevos análisis de regresión múltiple tomando como variables predictoras el optimismo y además de la satisfacción en los distintos dominios vitales. Los resultados indican que el optimismo tiene mayor capacidad predictiva que la satisfacción en áreas específicas, sobre todo en el caso de la felicidad. Concretamente, el 44\% de la felicidad $\left(F(5,252)=42.19 p<0.001, R^{2}\right.$ ajustado $=0.44$ ) fue predicha en primer lugar por el optimismo $(\beta=0.45, p<0.001)$, seguida de la satisfacción con los estudios $(\beta=0.22, p<0.001)$, la satisfacción con la pareja $(\beta=0.18, p<0.001)$, la satisfacción con el ocio $(\beta=0.11, p<0.01)$ y la satisfacción con la salud $(\beta=0.07, p<0.05)$. En el caso de la satisfacción vital, la incorporación del optimismo permitió predecir hasta un 30\% de la varianza $\left(F(5,252)=22.95 p<0.001, R^{2}\right.$ ajustado $=0.3)$ teniendo la misma importancia el optimismo $(\beta=0.23, p<0.001)$ que la satisfacción con los estudios $(\beta=0.23, p<0.001)$, seguidas de la satisfacción con la pareja $(\beta=0.20, p<0.001)$ y la satisfacción con el ocio $(\beta=0.10, p<0.01)$, pero no resultó significativa la satisfacción con la salud $(\beta=0.07, p=0.16)$.

\section{Discusión}

El objetivo de este trabajo era analizar la relación entre características sociodemográficas, optimismo y bienestar subjetivo, así como identificar la contribución de los dominios vitales y del optimismo al bienestar, en una población de universitarios de una institución mexicana. Los resultados indican

TABLA 4

Análisis de regresión de satisfacción en dominios vitales específicos sobre medidas de bienestar global

\begin{tabular}{lcccccc}
\hline & \multicolumn{3}{c}{ Satisfacción Vital } & \multicolumn{3}{c}{ Felicidad } \\
\hline \multicolumn{1}{c}{ Variables } & $\beta$ & $R^{2}$ adj & $F$ & $\beta$ & $R^{2}$ adj & $F$ \\
\hline Sastisfacción con estudios & $0.25^{* * *}$ & 0.25 & $22.86^{* * *}$ & $0.26^{* * *}$ & 0.26 & $23.41^{* * * *}$ \\
Satisfacción con pareja & $0.22^{* * *}$ & & & $0.22^{* * *}$ & & \\
Satisfacción con salud & $0.14^{* * *}$ & & & $0.14^{* *}$ & & \\
Satisfacción con ocio & $0.18^{*}$ & & & $0.18^{* *}$ & & \\
\hline
\end{tabular}

$* p<0.05 . * * p<0.01$. ***p $<0.001$.

Fuente: elaboración propia 
que las características sociodemográficas se asocian en menor medida al bienestar que el optimismo. La literatura previa ya había mostrado el escaso poder predictivo de las variables sociodemográficas sobre la felicidad y la satisfacción vital, explicando menos del 2\% de la varianza (Argyle, 1999; González, Moreno, Garrosa \& Peñacoba, 2005; Lykken \& Tellegen, 1996; Myers \& Diener, 1995; Watson, 2000).

En nuestro estudio, únicamente el género parecía guardar cierta relación con algunos indicadores de bienestar, presentando las mujeres mayor satisfacción con los estudios, con la vida y mayor felicidad que los hombres. En el metaanálisis de Wood, Rhodes y Whelan (1989) se encontró que las mujeres mostraban más felicidad que los hombres, posiblemente debido a que ellas experimentan tanto las emociones positivas como negativas con mayor intensidad (Larsen \& Diener, 1987). La mayor parte de los trabajos llevados a cabo con población mexicana corroboran estos resultados acerca del mayor bienestar de las mujeres frente a los hombres (Domínguez et al., 2006; Laca et al., 2005; Vera-Noriega \& Rodríguez, 2007). Probablemente, el hecho de que la mujer sea más sensible a las emociones y que informe de mayor empatía emocional que los hombres (Mestre, Samper, Frías $\&$ Tur, 2009) hace que se beneficie de otros factores que han aparecido vinculados al bienestar, como las relaciones positivas con los otros o el crecimiento personal (Ryff \& Keyes, 1995; Marrero \& Carballeira, 2012), y de ahí que valore su vida más positivamente. Además, la incorporación de la mujer al ámbito educativo y profesional en México es relativamente reciente y ha supuesto cambios en el papel que ella desempeña en la toma de decisiones familiares y personales (Casique, 2004; García \& Oliveira, 1994, 2001). Quizá esa creciente visibilidad de la mujer en la sociedad y haber incrementado su nivel de aspiraciones esté contribuyendo a que se produzca un empoderamiento de la mujer que haga que se perciba con un mayor control sobre su vida, lo que, a su vez, contribuiría de forma positiva a su bienestar (Casique, 2001).

Además, aunque la investigación previa había mostrado asociaciones entre estado civil y bienestar (Argyle, 1999; Myers \& Diener, 1995), en el presen- te estudio esta variable no presenta correlaciones significativas con el bienestar. Hay que tener en cuenta que la población universitaria es mayoritariamente soltera, en este caso más del 90\%, de ahí que esta variable no haya sido relevante. En su lugar, se analizó tener una relación sentimental que podría tomarse como un indicador del apoyo social con el que cuentan los individuos. Aunque solo un tercio de los participantes en esta investigación tenían pareja, este hecho sí parece estar asociado a la satisfacción en ese dominio concreto. Sin embargo, tener pareja no se relacionó con la felicidad o la satisfacción vital. Este es un hallazgo en el que se tendría que profundizar en estudios posteriores en los que, no solo se cuente con un mayor número de personas que estén implicadas en una relación sentimental sino que se valore la calidad de dicha relación, aspecto que parece estar más relacionado con el bienestar que el hecho en sí mismo de tener pareja (Diener et al., 1999).

Por otra parte, el optimismo resultó estar asociado a todos los indicadores de bienestar subjetivo, principalmente a felicidad y a emociones positivas, siendo la relación con la satisfacción en áreas específicas de menor magnitud. Las personas que se caracterizan por puntuar alto en optimismo también muestran una mayor puntuación en todos los indicadores de bienestar frente a las que se sitúan en posiciones intermedias o presentan bajo optimismo. Estos resultados son coherentes con los encontrados por Vera-Villarroel et al. (2009) que señalan la mayor satisfacción vital y felicidad de las personas con alto optimismo frente a las de bajo optimismo. Resulta relevante destacar que en nuestro estudio tener un nivel medio de optimismo es suficiente para que los individuos manifiesten mayor satisfacción vital, felicidad, emociones positivas, satisfacción con los estudios y con la salud y menor frecuencia de emociones negativas frente a los que presentan puntuaciones bajas en optimismo. Si además se incrementa el optimismo por encima de la media, podría tener repercusiones positivas sobre todo en el nivel de felicidad y en la satisfacción con áreas específicas, como la pareja y el ocio. Este resultado tiene importantes implicaciones a la hora de desarrollar programas de intervención que incrementen 
el optimismo como variable moduladora para promover el bienestar (Marrero \& Carballeira, 2010).

Por otra parte, también se encontraron relaciones significativas entre optimismo y género, informando las mujeres de un ligero mayor optimismo que los hombres. Una explicación tentativa para este resultado es que las mujeres optimistas, además de esperar resultados favorables, tal vez interpreten los acontecimientos con base en lo que esperan ganar o crecer mediante esas experiencias (Fry, 1995), lo que promueve que se generen emociones positivas y en última instancia el bienestar. De hecho, se ha encontrado que el apoyo emocional diario, junto con la sensación de autoeficacia, predicen el optimismo (Karademas, 2006).

Los resultados de este estudio indican, además, que el bienestar subjetivo es un constructo constituido por diferentes componentes claramente diferenciados, aunque relacionados. Las medidas globales, como la satisfacción vital y la felicidad, están más relacionadas con el componente afectivo (emociones positivas y negativas) que las medidas de satisfacción en dominios vitales específicos, resultados que son coherentes con los obtenidos en trabajos previos (Cummings et al., 2002). Aunque las emociones positivas también parecen estar implícitas en la valoración de la satisfacción con áreas específicas. La satisfacción, ya sea con la vida a nivel global o relativa a distintas áreas, supone integrar información diversa en la que posiblemente el individuo atienda al triple sistema de respuesta (cognitivo, emocional y comportamental), por lo que las emociones positivas van a jugar un papel importante en dicha valoración. Por tanto, la distinción de componentes emocionales y cognitivos, a la hora de analizar el bienestar, podría ser útil a nivel teórico, pero resulta "ficticia" a nivel aplicado, ya que el individuo va a analizar sus experiencias vitales de forma integral.

Por otra parte, la satisfacción vital no parece ser el resultado aditivo de la satisfacción en los dominios específicos, aunque guarda relación con los mismos. Los resultados obtenidos indican que la satisfacción en dominios específicos explica solo una cuarta parte de la varianza de satisfacción vital y de la felicidad. Los estudiantes que participaron en esta investigación han valorado la satisfacción con los estudios como el área que más contribuye a su satisfacción vital global y a su felicidad. Estudios previos han encontrado que la satisfacción con la familia y con los amigos se erigen como las áreas más relacionadas con la satisfacción vital y la felicidad (Banda \& Morales, 2012; Vera-Noriega, 2001). En nuestro estudio no se evaluaron directamente estos dominios, pero se incluyó una medida de satisfacción con la relación sentimental que recogía en cierto modo la importancia que tendrían los demás, concretamente la pareja, como fuente de apoyo y bienestar. Los resultados obtenidos indican que esta área tiene un peso importante en la satisfacción vital, más que la satisfacción con el ocio o con la salud, pero los estudios siguen siendo más relevantes para los universitarios, probablemente porque es una de las metas más prominentes que tiene este colectivo durante esta etapa vital. En trabajos posteriores, sería recomendable ahondar en estos resultados para determinar si la importancia de las áreas vitales puede variar a lo largo del tiempo en función de las demandas personales y del entorno.

En cualquier caso, el optimismo ha mostrado ser mejor predictor de la felicidad que la satisfacción en dominios específicos explicando conjuntamente más de un $40 \%$ de la varianza. Estudios previos avalan la relevancia del optimismo en la elección de estrategias de afrontamiento adaptativas (VeraVillarroel \& Guerrero, 2003; Wrosch, Scheier \& Miller, 2003), en la salud física (Juárez \& Landero, 2011; Seligman, 1998) o en el ajuste psicológico de los individuos y en su bienestar (Chang \& Sanna, 2001; Karademas, 2006; Marrero \& Carballeira, 2010).

Hay que tener en cuenta que los participantes de esta investigación eran estudiantes universitarios y trabajos previos indican que el nivel de bienestar de los mismos es superior al de personas adultas o de menor cualificación académica, ejerciendo la educación un cierto impacto en la satisfacción en los dominios laboral, familiar, familia extensa y amistad (Kamaruzaman, Zaliha, SoonYew \& Mohd Salleh, 2009; Laca \& Mejía, 2007; Luna, Laca \& Mejía, 2011; Rojas \& Elizondo-Lara, 2012; Salazar, 2009). Por tanto, los resultados de nuestro trabajo 
deben ser tomados con precaución y no pueden ser generalizados a otros grupos poblacionales.

Además, se han encontrado diferencias entre hombres y mujeres, tanto en su percepción del bienestar como en su nivel de optimismo, y en estudios con población anglosajona este tipo de diferencias no suelen manifestarse (Argyle, 1999; González et al., 2005; Marrero \& Carballeira, 2010), por lo que la cultura puede estar ejerciendo un papel en el modo en que se entiende el bienestar (Schimmack, Radhakrishnan, Oishi, Dzokoto \& Ahadi, 2002). Por tanto, en el futuro podría ser de interés analizar no solo si se mantienen las diferencias entre hombres y mujeres en felicidad, sino si existen diferencias intergénero en las fuentes y motivos de satisfacción, como han señalado algunos trabajos previos (Lu, 2000).

En suma, parece que las características sociodemográficas apenas inciden en el bienestar y que la satisfacción vital y la felicidad van más allá del efecto aditivo que supone estar satisfecho en distintos dominios de la vida, aunque para los universitarios que participaron en este estudio estar satisfechos con sus estudios contribuya en cierta medida a su satisfacción vital. Por su parte, el optimismo ha mostrado tener mayor peso a la hora de entender la felicidad de estos estudiantes, sin menoscabar la relevancia de la satisfacción con los estudios.

\section{Referencias}

Alarcón, R. (2001). Relaciones entre felicidad, género, edad y estado conyugal. Revista de Psicología de la PUCP, 19(1), 27-46.

Argyle, M. (1999). Causes and correlates of happiness. En D. Kahneman, E. Diener \& N. Schwarz (Eds.), Well-being: The foundations of hedonic psychology (pp. 353-373). New York: Russell Sage Foundation.

Arita, B. (2005). Satisfacción por la vida y teoría homeostática del bienestar. Psicología y Salud, 15(1), 121-126.

Banda, A. L. \& Morales, M. A. (2012). Calidad de vida subjetiva en estudiantes universitarios. Enseñanza e Investigación en Psicología, 17(1), 29-43.

Bradburn, N. M. (1969). The structure of psychological well-being. Chicago, IL: Aldin.
Brissette, I., Scheier, M. \& Carver, C. (2002). The role of optimism in social network development, coping, and psychological adjustment during a life transition. Journal of Personality and Social Psychology, 82(1), 102-111.

Casique I. (2001). Power, autonomy and division of labor in Mexican dual-earner families. Lanham, MD: University Press of America.

Casique, I. (2004). Poder de decisión y autonomía de la mujer mexicana. Análisis de algunos condicionantes. México: UNAM, Centro Regional de Investigaciones Multidisciplinarias.

Castro, A. (2012). La psicología positiva en América Latina. Desarrollos y perspectiva. Psiencia. Revista Latinoamericana de Ciencia Psicológica, 4(2), 108-116.

Casullo, M. M. (2000). Psicología salugénica o positiva. Algunas reflexiones. Anuario de Investigaciones, 8 , 340-346.

Chang, E. C. \& Sanna, L. J. (2001). Optimism, pessimism, and positive and negative affectivity in middle-aged adults: A test of a cognitive-affective model of psychological adjustment. Psychology and Aging, 16(3), 524-531.

Chico, E. (2002). Optimismo disposicional como predictor de estrategias de afrontamiento. Psicothema, 14(3), 544-550.

Cummings, R., Eckersley, J., Pallant, J. \& Davern, M. (2002). The international wellbeing group and Australian unity well-being index (Survey 3: Report 1). Melbourne: Australian Centre on Quality of Life/ Deakin University.

DeNeve, K. M. \& Cooper, H. (1998). The happy personality: A meta-analysis of 137 personality traits and subjective well-being. Psychological Bulletin, 124(2), 197-229.

Díaz, F. \& González, M. J. (2011). Subjective and occupational well-being in a sample of Mexican workers. Social Indicators Research, 100(2), 273-285.

Diener, E. (1984). Subjective well-being. Psychological Bulletin, 95(3), 542-575.

Diener, E., Emmons, R. A., Larsen, R. J. \& Griffin, S. (1985). The Satisfaction with Life Scale. Journal of Personality Assessment, 49(1), 71-75. 
Diener, E., Suh, E. M., Lucas, R. E. \& Smith, H. L. (1999). Subjective well-being: Three decades of progress. Psychological Bulletin, 125(2), 276-302.

Domínguez M. T., Batista F. J., Tôrres, B., Vera, J. A., Becerra, M. A. \& Domínguez R. L. (2006). Relação do bem-estar subjetivo, estratégias de enfrentamento e apoio social em idosos. Psicologia: Reflexão e Crítica, 19(2), 301-308.

Escobar, Y., Zanatta, E., Ponce, T., García, S. L. \& Gama, J. L. (2012). Identidad y bienestar psicológicos: estilos y factores en estudiantes universitarios. Revista de Psicología Nueva Época 2, 1(1), 41-53.

Fordyce, M. W. (1986). The Psychap Inventory: A multiscale test to measure happiness and its concomitants. Social Indicarors Research, 18(1), 1-33.

Fry, P. S. (1995). Perfectionism, humor, and optimism as moderators of health outcomes and determinants of coping styles of women executives. Genetics, Social, and General Psychology Monographs, 121(2), 211-245.

Fuentes, N. \& Rojas, M. (2001). Economic theory and subjective well-being: Mexico. Social Indicators Research, 53(1), 34-41.

García, B. \& Oliveira, O. (1994). Trabajo femenino y vida familiar en México (Vol. 11). México: Centro de Estudios Demográficos y de Desarrollo Urbano.

García, B. \& Oliveira, O. (2001). Transformaciones recientes en los mercados de trabajo metropolitanos de México: 1990-1998. Estudios Sociológicos, 19(57), 653-689.

González, J. L., Moreno, B., Garrosa, E. \& Peñacoba, C. (2005). Personality and subjective well-being: Big Five correlates and demographic variables. Personality and Individual Differences, 38(7), 1561-1569.

Juárez, D. \& Landero. R. (2011). Imagen corporal, funcionamiento sexual, autoestima y optimismo en mujeres con cáncer de mama. Revista Electrónica Nova Scientia, 4(7), 17-34.

Kamaruzaman, J., Zaliha H. H., SoonYew, J. \& Mohd Salleh, H. D. (2009). The life satisfaction of academic and non-academic staff in a Malaysian higher education institution. International Education Studies, 2(1), 143-150.

Karademas, E. C. (2006). Self-efficacy, social support and well-being: The mediating role of opti- mism. Personality and Individual Differences, 40(6), 1281-1290.

Laca, F. A. \& Mejía. J. C. (2007). Actitudes ante la violencia, bienestar subjetivo e individualismo en jóvenes mexicanos. Enseñanza e Investigación en Psicología, 12(2), 301-313.

Laca, F. A., Verdugo, J. C. \& Guzmán, J. (2005). Satisfacción con la vida de algunos colectivos mexicanos: una discusión sobre la psicología del bienestar subjetivo. Enseñanza e Investigación en Psicología, $10(2), 325-336$.

Larsen, R. J. \& Diener, E. (1987). Affect intensity as an individual difference characteristic: A review. Journal of Research in Personality, 21(1), 1-39.

Lu, L. (2000). Gender and conyugal differences in happiness. The Journal of Social Psychology, 140(1), 132-141.

Luna, A. C., Laca, F. A. \& Mejía, J. C. (2011). Bienestar subjetivo y satisfacción con la vida de familia en adolescentes mexicanos de bachillerato. Psicología Iberoamericana, 19(2), 17-26.

Lykken, D. \& Tellegen, A. (1996). Happiness is a stochastic phenomenon. Psychological Science, 7(3), 186-189.

Lyubomirsky, S. \& Lepper, H. S. (1999). A measure of subjective happiness: Preliminary reliability and construct validation. Social Indicators Research, 46(2), 137-155.

Marrero, R. J., Carballeira, M. \& Rodríguez, J. P. (2007). El papel del optimismo en la salud (Memoria final de investigación). Proyecto subvencionado por el Vicerrectorado de Investigación y Desarrollo Tecnológico de la Universidad de La Laguna, Tenerife, España.

Marrero, R. J. \& Carballeira, M. (2010). El papel del optimismo y del apoyo social en el bienestar subjetivo. Salud Mental, 33(1), 39-46.

Marrero, R. J. \& Carballeira, M. (2011). Well-being and personality: Facet-level analyses. Personality and Individual Differences, 50(2), 206-211.

Marrero, R. J. \& Carballeira, M. (2012). The contribution of personality to PWB: An exploratory study. En B. S. Nguyem (Ed.), Psychology of satisfaction (pp 41-65). New York: NOVA.

Mestre, M. V., Samper, P., Frías, M. D. \& Tur, A. M. (2009). Are women more empathetic than men? 
A longitudinal study in adolescence. The Spanish Journal of Psychology, 12, 63-83.

Moyano, E. \& Ramos, N. (2007). Bienestar subjetivo: midiendo satisfacción vital, felicidad y salud en población chilena de la región del Maule. Revista Universum, 22(2), 185-200.

Myers, D. G. \& Diener, E. (1995). Who is happy? Psychological Science, 6(1), 10-19.

Namkee, A. \& Mochón, F. (2007, mayo). La felicidad de los españoles: factores explicativos (Documento de trabajo № 2007-12). Fundación de Estudios de Economía Aplicada. Recuperado de http://www. fedea.es/pub/papers/2007/dt2007-12.pdf

Palomar, J. (2000). The development of an instrument to measure quality of life in Mexico City. Social Indicators Research, 50(2), 187-208.

Palomar, J. (2004). Poverty and subjective well-being in Mexico. Social Indicators Research, 68(1), 1-33.

Pavot, W. \& Diener, E. (1993). Review of the Satisfaction with Life Scale. Psychological Assessment, 5(2), 164-172.

Puente-Díaz, R. \& Cavazos, J. (2013). Personality factors, affect, and autonomy support as predictors of life satisfaction. Universitas Psychologica, 12(1), 41-53.

Rojas, M. \& Elizondo-Lara, M. (2012). Satisfacción de vida en Costa Rica: un enfoque de dominios de vida. Latin American Research Review, 47(1), 78-94.

Ruvalcaba, N. A., Salazar, J. G. \& Fernández-Berrocal, P. (2012). Indicadores sociales, condiciones de vida y calidad de vida en jóvenes mexicanos. Revista Iberoamericana de Psicología: Ciencia y Tecnología, 5(1), 71-80.

Ryan, R. M. \& Deci, E. L. (2001). On happiness and human potentials: A review of research on hedonic and eudaimonic well-being. Annual Review of Psychology, 52(1), 141-166.

Ryff, C. D. (1989). Happiness is everything, or is it? Explorations on the meaning of psychological wellbeing. Journal of Personality and Social Psychology, 57(6), 1069-1081.

Ryff, C. D. \& Keyes, C. L. M. (1995). The structure of PWB revisited. Journal of Personality and Social Psychology, 69(4), 719-727.
Salazar, F. A. (2009). Felicidad en estudiantes de la Facultad de Salud de la Universidad Surcolombiana. Revista Facultad de Salud, 1(2), 17-23.

Scheier, M. F. \& Carver, C. S. (1985). Optimism, coping and health: Assessment and implications of generalized outcome expectancies. Health Psychology, 4(3), 219-247.

Scheier, M. F., Carver, C. S. \& Bridges, M. W. (1994). Distinguishing optimism from neuroticism (and trait anxiety, self-mastery, and self-esteem): A reevaluation of the Life Orientation Test. Journal of Personality and Social Psychology, 67(6), 1063-1078.

Schimmack, U., Radhakrishnan, P., Oishi, S., Dzokoto, V. \& Ahadi, S. (2002). Culture, personality, and subjective well-being: Integrating process models of life satisfaction. Journal of Personality and Social Psychology, 82(4), 582-593.

Seligman, M. (1998). Aprenda optimismo. Haga de la vida una experiencia maravillosa. Barcelona: Grijalbo.

Seligman, M. E. P. \& Csikszentmihalyi, M. (2000). Positive psychology: An introduction. American Psychologist, 55(1), 5-14.

Steel, P., Schmidt, J. \& Shultz, J. (2008). Refining the relationship between personality and subjective well-being. Psychological Bulletin, 134(1), 138-161.

Vera-Noriega, J. A. (2001). Bienestar subjetivo en una muestra de jóvenes universitarios. Revista Intercontinental de Psicología y Educación, 3(1), 11-21.

Vera-Noriega, J. A. \& Rodríguez, E. J. (2007). La felicidad y sus correlatos en los estudiantes de la Universidad de Sonora. Revista de la Universidad de Sonora, 19, 17-19.

Vera-Noriega, J. A. \& Tánori, B. C. (2002). Propiedades psicométricas de un instrumento para medir bienestar subjetivo en la población mexicana. Apuntes de Psicología, 20(1), 63-80.

Vera-Villarroel, P. \& Guerrero, A. (2003). Diferencias en habilidades de resolución de problemas sociales en sujetos optimistas y pesimistas. Universitas Psychologica, 2(1), 21-26.

Vera-Villarroel, P., Córdova-Rubio, N. \& Celis-Atenas, K. (2009). Optimismo versus autoestima: implicancia para la psicología clínica y psicoterapia. Revista Argentina de Clínica Psicológica, 18(1), 21-30. Verdugo-Lucero, J. L., Ponce de León-Pagaza, B. G., Guardado-Llamas, R. E., Meda-Lara, R. M., Uribe- 
Alvarado, J. I. \& Guzmán-Muñiz, J. (2013). Estilos de afrontamiento al estrés y bienestar subjetivo en adolescentes y jóvenes. Revista Latinoamericana de Ciencias Sociales, Niñez y Juventud, 11(1), 79-91.

Watson, D. (2000). Mood and temperament. New York: Guilford.

Watson, D., Clark, L. A. \& Tellegen, A. (1988). Development and validation of brief measures of positive and negative affect: The PANAS Scales. Journal of Personality and Social Psychology, 54(6), 1063-1070.
Wood, W., Rhodes, N. \& Whelan, M. (1989). Sex differences in positive well-being: A consideration of emotional style and marital status. Psychological Bulletin, 106(2), 249-264.

Wrosch, C., Scheier, M. F. \& Miller, G. E. (2003). Adaptive self-regulation of unattainable goals: Goal disengagement, goal reengagement, and subjective well-being. Personality and Social Psychology Bulletin, 29(12), 1494-1508. 